\title{
Contextual Argument Component Classification for Class Discussions
}

\author{
Luca Lugini and Diane Litman
}

\author{
Department of Computer Science and \\ Learning Research and Development Center \\ University of Pittsburgh \\ Pittsburgh, PA, USA
}

\begin{abstract}
Argument mining systems often consider contextual information, i.e. information outside of an argumentative discourse unit, when trained to accomplish tasks such as argument component identification, classification, and relation extraction. However, prior work has not carefully analyzed the utility of different contextual properties in context-aware models. In this work, we show how two different types of contextual information, local discourse context and speaker context, can be incorporated into a computational model for classifying argument components in multi-party classroom discussions. We find that both context types can improve performance, although the improvements are dependent on context size and position.
\end{abstract}

\section{Motivation}

In a typical argument mining system, the first task is identifying spans of text consisting of argumentative discourse units (ADU), i.e. argument component identification (ACI). The next task, argument component classification (ACC), consists of assigning a label to each ADU according to an argument model, e.g. claims, evidence, etc. For example, row 1 in Table 1 is labeled claim since speaker 7 provides their personal view, while row 2 is labeled evidence because it references facts from a text.

While "context" has been used in the argument mining literature to refer to several phenomena, we consider context to be auxiliary textual information outside the span of an ADU. It is generally accepted that context is important in argument mining. Stab and Gurevych (2014) as well as Nguyen and Litman (2016) use context features extracted from the sentence containing an ADU to improve ACC. Persing and $\mathrm{Ng}$ (2016), Habernal and Gurevych (2017) and Aker et al. (2017) similarly use contextual features in joint ACI/ACC models. Optiz and Frank (2019) analyze a previous argument mining system and find that, for its predictions, it relies on context more than it does on ADU content. Chakrabarty et al. (2019) indirectly model context in ACC by fine-tuning a BERT model to predict the next sentence (i.e. the context). Eger et al. (2017) analyzed several neural models for jointly performing ACI, ACC and argument relation extraction. All of these works share several limitations: (i) the context is either limited to a single configuration (e.g. one sentence before/after the ADU) or optimized along a single dimension (typically size but not position); (ii) only a subset of the features extracted for the target ADU are also extracted for context; (iii) context is typically based on ADU adjacency, although other ways of building context (e.g. based on speakers in multi-party dialogues) are possible.

\begin{tabular}{|c|c|c|c|}
\hline Row & ID & ADU & $\mathbf{A C}$ \\
\hline 1 & 7 & I feel that she included herself in the book to give more of an unbiased point of view to it. & claim \\
\hline 2 & 7 & $\begin{array}{l}\text { Because, obviously the family was going to be completely against the doctors and what they did, } \\
\text { and all that. }\end{array}$ & evidence \\
\hline 3 & 7 & But I feel by her including herself in it, she did show both sides of the story. & warrant \\
\hline 4 & 10 & $\begin{array}{l}\text { I'd argue against that keeping her as unbiased. Because I think it puts more of a bias on the Lacks } \\
\text { family's side. }\end{array}$ & claim \\
\hline 5 & 7 & $\begin{array}{l}\text { I also feel she included herself because this was such a big part of her career from learning all } \\
\text { this. And she became attached to the family members. }\end{array}$ & claim \\
\hline
\end{tabular}

Table 1: Excerpt from a classroom discussion showing speaker ID, ADU and argument component labels. 
In this work, we improve upon baseline ACC models for multi-party discussions by incorporating two types of contexts. We define local context as ADUs preceding and/or following a target ADU, regardless of speaker. Speaker context consists of previous ADUs that a specific speaker previously voiced during the discussion. Our results show that both context types can individually and jointly improve ACC performance, with performance gains dependent on context size and position.

\section{Dataset and Models}

The dataset ${ }^{1}$ used to build and evaluate our proposed ACC models consists of 3,135 ADUs in a corpus of 29 text-based (i.e. centered around a book, play, or other literature piece), multi-party classroom discussions between high school students (average 15 students per discussion) (Olshefski et al., 2020). The discussions (average length of 34 minutes) were audio-recorded, manually transcribed, and student turns were manually segmented into multiple ADUs when needed. ADUs were then manually annotated according to a simplified version of Toulmin's argumentative model (Toulmin, 1958) consisting of three labels: (i) claims, arguable statements that voice a specific interpretation of a text; (ii) evidence, facts or documentation used to support a claim; (iii) warrant, reasoning given to explain how certain evidence supports a claim. An inter-rater reliability analysis showed a Krippendorff $\alpha_{U}$ of 0.96 for segmentation and Cohen Kappa of 0.74 for argument components. The dataset contains 3,135 ADUs: $65.3 \%$ claims, $24.3 \%$ evidence, and $10.4 \%$ warrants. ADUs are additionally labeled with the ID of the speaker who voiced the utterance. Table 1 shows an excerpt from an annotated discussion.

To evaluate the utility of context, we introduce two baseline ACC models and propose several contextual extensions. The source code for all models (and all parameters) is available at https: //github.com/lucalugini/coling2020_argmining.

Baseline Models. Our first model (hybrid baseline) is based on the model of Lugini and Litman (2018) which was developed for a similar type of dataset, where an embedding generated through a convolutional neural network $(\mathrm{CNN})$ is concatenated to a set of handcrafted features, and a softmax classifier is used to predict argument components. Given the limited size of our dataset, however, we only use a subset of the original model's handcrafted features, namely those used by Speciteller (Li and Nenkova, 2015); this reduces the number of handcrafted features from over 7,000 to 114 and avoids overfit. The Speciteller feature set consists of pretrained word vectors (average of the word vectors for each word in the ADU), as well as number of connectives, number of words, number of numbers, number of symbols, number of capital letters, number of stopwords normalized by ADU length, number of subjective and polar words (from the MPQA (Wilson et al., 2009) and the General Inquirer (Stone and Hunt, 1963) dictionaries), average word familiarity (from MRC Psycholinguistic Database (Wilson, 1988)), average characters per word, and inverse document frequency statistics (minimum and maximum). The dimensionality of the final feature vector is 2,514 (114 for the handcrafted features and 2,400 for the CNN). Our second model (BERT baseline) is based on recent advances related to Transformer architectures (Vaswani et al., 2017): a BERT pretrained model (Devlin et al., 2019; Wolf et al., 2019) generates word embeddings of dimensionality 768; average pooling is used to compute a fixed-size ADU embedding; a softmax classifier predicts argument components.

Adding Local Context. We define local context as ADUs preceding and/or following the target ADU, regardless of speaker ID. Context size is measured in terms of complete ADUs (i.e. entire utterance or part of it), while context position refers to the relative position of the context ADUs to the target ADU (i.e. preceding, following, both). We believe defining context in terms of ADUs is the most straightforward choice since it is the same unit of analysis used for individual argument components. Though beyond the scope of this paper, another compelling choice consists in defining context based on the number of words outside the target ADU instead. We address the prior work limitations highlighted earlier in two ways: $(i)$ we explore the impact of varying both the size and position of ADUs included in the context, instead of picking a single position and optimizing size; (ii) we model context using the same features used for the target ADU. Each context ADU is converted into a fixed-size feature vector using the baseline models described above, then concatenated to the feature vector for the target ADU. A

\footnotetext{
${ }^{1}$ Obtained from https://discussiontracker.cs.pitt.edu
} 
maximum context size of 6 was chosen based on results showing diminishing returns and on the fact that increasing size further would go beyond "local" context. We additionally evaluate whether context size and position can be automatically optimized by adding an attention layer (Luong et al., 2015): context size is set to the maximum value and both preceding and following positions are included; the attention mechanism then aggregates all context ADUs into a single vector.

Adding Speaker Context. Students exhibit highly variable behavior with respect to how they build arguments. For example, in a discussion between six students from the dataset, one student only voiced claims, only two students voiced warrants, and only two students voiced more than $10 \%$ of their argument components as evidence. We hypothesize that the argument component classifier can benefit from being informed of the propensity of a particular speaker to voice each argument component at any given point. While we have access to speaker ID, when making predictions ground truth ADU labels are not available; therefore we need to extract information from the ADU text. Given the speaker ID for the target ADU, the speaker context module performs the following steps: (1) gather the speaker's previous ADUs from the discussion; (2) convert each ADU into a feature vector; (3) aggregate them into a single, fixed-size feature vector and concatenate it with the baseline (and possibly with the local context). Step 1 involves simply filtering out ADUs based on speaker ID, which is readily available in each discussion. Step 2 can be achieved in several ways, however, for the sake of simplicity, in the hybrid baseline we decided to use a CNN to generate a feature vector for each ADU. In order to further reduce complexity we implemented a CNN with the same structure as the one in the hybrid baseline model, but with the number of filters reduced from 16 to 4 . This resulted in a 200-dimensional vector. For the BERT baseline the same embedding - average pooling model was used in this step. Step 3 was accomplished using a Long ShortTerm Memory (LSTM) network (Hochreiter and Schmidhuber, 1997). The final speaker context feature vector has dimensionality 100 (output dimensionality of the LSTM). We additionally experimented with automatically optimizing speaker context size by replacing the LSTM with an attention layer: setting the speaker context size to the maximum (40 in this case) the attention layer aggregates all ADUs into a single feature vector.

Context Examples. Assume the target ADU is row 5 in Table 1. A local context of size 3 contains rows 2, 3, and 4. A speaker context of size 3 contains rows 1, 2 and 3.

\section{Experiments and Results}

All models are evaluated using ten fold cross-validation, and results are shown in Table 2.

Local Context. We extended both baseline models with local context extracted in three different ways: only ADUs preceding the target, only following ADUs, and both preceding/following ADUs. We report three main observations when adding context to the hybrid baseline. First, with respect to position, all models including prior ADUs in local context significantly outperformed the baseline (pvalue $<0.01$ ), while the same is not true for models including next ADUs. Including both prior and next ADUs, though, resulted in the best performance for local context models (row 2 in Table 2). When including only next ADU context, only context size 1 gave a significant performance improvement over the baseline, while larger context sizes yielded non statistically significant differences. Second, with respect to context size, the plots in Figure 1 (a) show that although there is a diminishing return effect as

\begin{tabular}{|c|c|c|c|c|c|c|}
\hline Row & Model & Context & Kappa & Precision & Recall & F-score \\
\hline 1 & \multirow{4}{*}{ Hybrid Baseline } & - & 0.350 & 0.535 & 0.531 & 0.509 \\
\hline 2 & & Local Context & 0.521 & 0.657 & 0.727 & 0.676 \\
\hline 3 & & Speaker Context & 0.470 & 0.626 & 0.682 & 0.636 \\
\hline 4 & & Local Context + Speaker Context & $\mathbf{0 . 5 3 9}$ & 0.674 & 0.727 & 0.689 \\
\hline 5 & \multirow{4}{*}{ BERT Baseline } & - & 0.483 & 0.620 & 0.669 & 0.632 \\
\hline 6 & & Local Context & 0.657 & 0.759 & 0.787 & 0.769 \\
\hline 7 & & Speaker Context & 0.625 & 0.733 & 0.794 & 0.751 \\
\hline 8 & & Local Context + Speaker Context & 0.653 & 0.759 & $\mathbf{0 . 8 1 0}$ & $\mathbf{0 . 7 7 4}$ \\
\hline
\end{tabular}

Table 2: Results for different experimental settings. Each row shows the best results for the corresponding settings when varying context size and position. Bold font shows the best results for each model. 


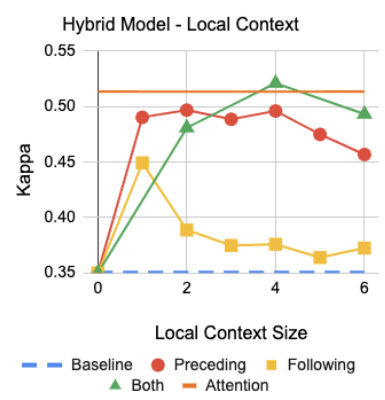

(a)

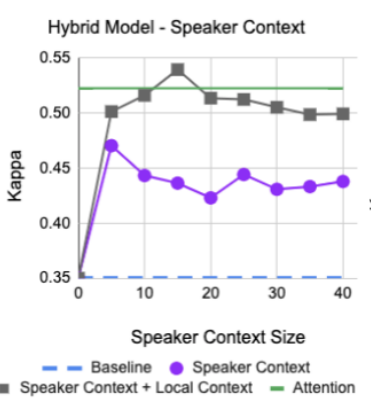

(b)

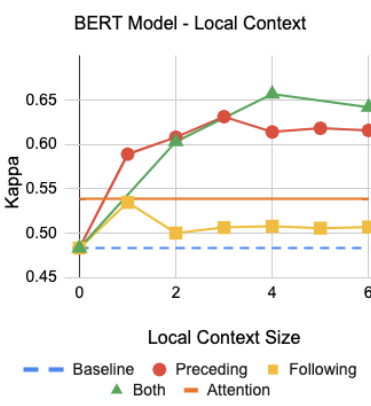

(c)

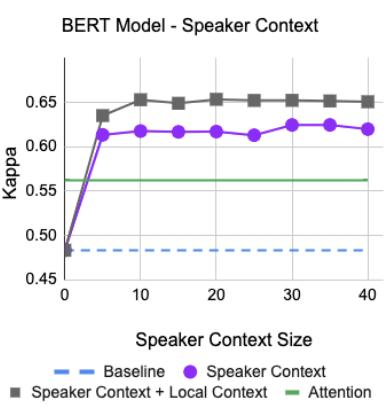

(d)

Figure 1: Results for various context sizes. (a) and (c) compare the baselines to models with local context. (b) and (d) compare the baselines with speaker context and both context types.

context size increases, increasing context size from 2 ( 1 prior/next ADU) to 4 ( 2 prior/next ADUs) results in significantly better precision and f-score (p-value $<0.05$ ). Overall, we argue that context position and size should both be optimized. Figure 1 (a) and (c) show that choosing the optimal context position may even be more important than choosing the optimal size: the differences between the three lines are bigger than differences within each line. Also note that since model complexity increases linearly with context size, larger datasets may be able to take advantage of larger contexts. Third, results obtained using the attention mechanism are not statistically significantly different than the best results for both local context and speaker context. This indicates that context size and position can be automatically optimized during training time with a marginal performance penalty. The results obtained from the BERT models confirm our first two observations, as we can see from the similarities in Figures 1 (a) and (c). For the BERT models, however, the attention mechanism performs significantly worse than any setting that includes at least 1 preceding ADU. In this case context size and position still need to be tuned hyperparameters. We should also note that overall BERT models perform better than the hybrid models.

Speaker Context. We define speaker context size $\mathrm{K}$ as the $\mathrm{K}$ closest prior ADUs to the target ADU. We experimented with speaker context sizes ranging from a minimum of 5 to a maximum of 40 (effectively considering all speaker turns since the beginning of the discussion). As shown in Figure 1 (b), including any previous ADUs from the current speaker improves performance over the baseline model. For hybrid models all context sizes result in statistically significantly better precision, recall, and $\mathrm{f}$-score ( $\mathrm{p}$-value $<0.05$ ) compared to the baseline. We observed a diminishing return effect as speaker context size increases as well, which suggests that a speaker's recent ADUs are perhaps more important than earlier ones. By comparing the green and red lines in Figure 1 (a) to the purple line in Figure 1 (b) we observed that, taken individually, local context improves performance more than speaker context. Results also show that the attention mechanism can successfully optimize speaker context size for the hybrid model. Figure 1 (d) shows that our observations for BERT models are largely consistent with those for hybrid models, though we note that BERT models seem less sensitive to speaker context size. Unfortunately, adding attention mechanisms to optimize speaker context size results in a large performance penalty, though still outperforming the baseline model.

Local Context and Speaker Context. After individually adding each of the two context types to the baseline models, we experimented with including both context types simultaneously. In this setting we obtained the best overall performance (rows 4 and 8 of Table 2). As we can see from the grey and purple lines in Figure 1 (b), for hybrid models, modeling both contexts simultaneously always outperforms speaker context alone. For speaker context size $>5$, improvements are statistically significant. In this experiment we kept local context size and position constant (both prior and next ADUs, size 4) and varied speaker context size. Repeating this experiment with different local context settings yielded similar results. We also observed that by combining both context types we were able to achieve kappa $>0.5$ and f1-score $>0.65$ more consistently than with either context type individually. We observed consistent trends for BERT models, although the differences between speaker context alone and local context + speaker context are often not statistically significant. 


\section{Conclusions and Future Work}

In this paper we analyzed the impact of context for predicting argument components in multi-party discussions. We defined two types of context, local context and speaker context, and analyzed how different models perform when varying context size. We also investigated the effect of different positions for local context. We performed evaluations of the two context types separately as well as simultaneously on two types of neural network models. Experimental results support our claim that both context size and position are important when incorporating context in argument mining systems, therefore sentences beyond the ones immediately surrounding an ADU should be considered. Our results also show that speaker context can improve performance in multi-party discussions. Finally, we investigated the use of an attention mechanism for optimizing context size and found that its effectiveness is dependent on the type of model used. Our future plans include two main directions: 1) repeating our local context experiments on larger datasets, including other domains; 2) evaluating the effectiveness of speaker context on multi-party web discussions, where discussions are usually longer and author ID is typically available.

\section{Acknowledgements}

This work was supported by the National Science Foundation (1842334 and 1917673), and in part by the University of Pittsburgh Center for Research Computing through the resources provided.

\section{References}

Ahmet Aker, Alfred Sliwa, Yuan Ma, Ruishen Lui, Niravkumar Borad, Seyedeh Ziyaei, and Mina Ghobadi. 2017. What works and what does not: Classifier and feature analysis for argument mining. In Proceedings of the 4th Workshop on Argument Mining, pages 91-96.

Tuhin Chakrabarty, Christopher Hidey, Smaranda Muresan, Kathy McKeown, and Alyssa Hwang. 2019. AMPERSAND: Argument mining for PERSuAsive oNline discussions. In Proceedings of the 2019 Conference on Empirical Methods in Natural Language Processing and the 9th International Joint Conference on Natural Language Processing (EMNLP-IJCNLP), pages 2933-2943, Hong Kong, China, November.

Jacob Devlin, Ming-Wei Chang, Kenton Lee, and Kristina Toutanova. 2019. BERT: Pre-training of deep bidirectional transformers for language understanding. In Proceedings of the 2019 Conference of the North American Chapter of the Association for Computational Linguistics: Human Language Technologies, pages 4171-4186, Minneapolis, Minnesota, June.

Steffen Eger, Johannes Daxenberger, and Iryna Gurevych. 2017. Neural end-to-end learning for computational argumentation mining. In Proceedings of the 55th Annual Meeting of the Association for Computational Linguistics (Volume 1: Long Papers), pages 11-22.

Ivan Habernal and Iryna Gurevych. 2017. Argumentation mining in user-generated web discourse. Computational Linguistics, 43(1):125-179.

Sepp Hochreiter and Jürgen Schmidhuber. 1997. Long short-term memory. Neural computation, 9(8):1735-1780.

Junyi Jessy Li and Ani Nenkova. 2015. Fast and accurate prediction of sentence specificity. In Proceedings of the Twenty-Ninth Conference on Artificial Intelligence (AAAI), pages 2281-2287, January.

Luca Lugini and Diane Litman. 2018. Argument component classification for classroom discussions. In Proceedings of the 5th Workshop on Argument Mining, pages 57-67.

Minh-Thang Luong, Hieu Pham, and Christopher D Manning. 2015. Effective approaches to attention-based neural machine translation. In Proceedings of the 2015 Conference on Empirical Methods in Natural Language Processing, pages 1412-1421.

Huy Nguyen and Diane J Litman. 2016. Improving argument mining in student essays by learning and exploiting argument indicators versus essay topics. In FLAIRS Conference, pages 485-490.

Christopher Olshefski, Luca Lugini, Ravneet Singh, Diane Litman, and Amanda Godley. 2020. The discussion tracker corpus of collaborative argumentation. In Proceedings of The 12th Language Resources and Evaluation Conference, pages 1033-1043, Marseille, France, May. European Language Resources Association. 
Juri Opitz and Anette Frank. 2019. Dissecting content and context in argumentative relation analysis. In Proceedings of the 6th Workshop on Argument Mining, pages 25-34, Florence, Italy, August.

Isaac Persing and Vincent Ng. 2016. End-to-end argumentation mining in student essays. In Proceedings of the 2016 Conference of the North American Chapter of the Association for Computational Linguistics: Human Language Technologies, pages 1384-1394.

Christian Stab and Iryna Gurevych. 2014. Annotating argument components and relations in persuasive essays. In Proceedings of COLING 2014, the 25th International Conference on Computational Linguistics: Technical Papers, pages 1501-1510.

Philip J Stone and Earl B Hunt. 1963. A computer approach to content analysis: studies using the general inquirer system. In Proceedings of the May 21-23, 1963, spring joint computer conference, pages 241-256. ACM.

Stephen Toulmin. 1958. The uses of argument. Cambridge: Cambridge University Press.

Ashish Vaswani, Noam Shazeer, Niki Parmar, Jakob Uszkoreit, Llion Jones, Aidan N Gomez, Łukasz Kaiser, and Illia Polosukhin. 2017. Attention is all you need. In Advances in neural information processing systems, pages 5998-6008.

Theresa Wilson, Janyce Wiebe, and Paul Hoffmann. 2009. Recognizing contextual polarity: An exploration of features for phrase-level sentiment analysis. Computational linguistics, 35(3):399-433.

Michael Wilson. 1988. Mrc psycholinguistic database: Machine-usable dictionary, version 2.00. Behavior Research Methods, 20(1):6-10.

Thomas Wolf, Lysandre Debut, Victor Sanh, Julien Chaumond, Clement Delangue, Anthony Moi, Pierric Cistac, Tim Rault, R'emi Louf, Morgan Funtowicz, and Jamie Brew. 2019. Huggingface's transformers: State-of-theart natural language processing. ArXiv, abs/1910.03771. 